\title{
Effects of Standard Coagulant Agents on the Dielectric Properties of Fresh Human Blood
}

\author{
Saqib Salahuddin, Martin O'Halloran, Emily Porter \\ National University of Ireland Galway \\ Department of Electrical and Electronic Engineering \\ Galway, Ireland \\ Lourdes Farrugia, Julian Bonello, Charles V. Sammut \\ University of Malta \\ Department of Physics \\ Msida, Malta \\ and P. Schembri Wismayer \\ University of Malta \\ Department of Anatomy \\ Msida, Malta
}

\begin{abstract}
In this paper, the effects of coagulation and temperature on the dielectric properties of human blood are investigated over the frequency range of $400 \mathrm{MHz}-20 \mathrm{GHz}$ using freshly extracted blood samples. The dielectric properties are measured using blood in four different sample collection tubes (bottles): one containing pure whole blood, two containing different anticoagulant agents, and one containing clot activator and serum separator. The collected data indicates that additive agents can have a significant impact on the measured dielectric properties of blood, both immediately after the sample is taken, and over longer time periods. This is an important finding as it suggests that measurements of blood properties conducted on sample repositories, or tissue banks, may not be representative of natural blood properties. Further, the results demonstrate that the dielectric properties of normal blood vary over time due to coagulation. Different clotting rates lead to dielectric properties of female and male blood samples that vary distinctly over time. The results also show that the relative permittivity of the anti-coagulated blood decreases with increasing temperature, up to the cross-over point around $10 \mathrm{GHz}$ where the trend reverses.
\end{abstract}

Index Terms - Dielectric measurements, biological material, Human blood, Coagulation, relative permittivity.

\section{INTRODUCTION}

BLOOD is a complex and highly functional fluid in humans and other animals. The blood serves as the principle transport medium responsible for delivering the necessary substances such as nutrients, oxygen and vitamins, to vital parts of the body. It also transports metabolic waste products away from cells making it a fundamental part of the immune system [1]. Precise knowledge of the constituents of blood, and its physical, biological and chemical properties, is of great importance. In particular, the dielectric properties of human blood are capable of revealing a variety of dynamic processes and are vitally important in modelling of biological tissues with respect to their in vivo

Manuscript received on 5 January 2017, in final form 11 April 2017, accepted 18 July 2017. Corresponding author: S. Salahuddin. characteristics.

The dielectric properties of blood are extremely significant in a multitude of medical applications. These properties are used in cell separation of cancer cells from normal blood cells [2]. Dielectric spectroscopy is used to examine the deterioration of preserved blood [3]. Another application of dielectric properties of blood is dielectric coagulometry. It can assess platelet function, fibrin formation, fibrinolysis and the anticoagulant effect of heparin [4]. Further, the dielectric response of blood can be used to estimate the venous thrombosis risk [5] which is the third cause of excess mortality in U.S. hospitals [6]. The dielectric properties also determine the pathways of current flow through the human body and are very important in the analysis of a wide range of biomedical applications such as functional electrical stimulation, 
the diagnosis and treatment of various physiological conditions with weak electric current currents, radio-frequency hyperthermia and ablation, body composition, and electrocardiography [7][14]. Moreover, precise knowledge of the dielectric properties of blood is essential for determination and measurement of safe limits for electromagnetic energy absorption by the human body [15]-[17].

Several research studies have been performed on the dielectric properties of biological fluids [18]-[22]. In the past two decades, most published papers on the dielectric spectroscopy of blood and erythrocyte solutions have addressed specific aspects such as electrical impedance, erythrocyte suspension, polarization effect, and hematocrit dependency [23]-[34]. So far, the most cited work reporting the broadband spectra of blood covering several dispersion regions is by Gabriel et al. [35], taken at $37^{\circ} \mathrm{C}$, which is commonly used for specific absorption rate (SAR) calculations and medical purposes. However, the available data is limited in scope. It does not address changes due to temperature or interpatient and inter-gender variability. Furthermore, the coagulation is not controlled for and the effect of clotted versus un-clotted blood on the dielectric properties is not investigated.

Despite the research done so far, many aspects of the dielectric properties of blood are still unclear. To best of authors' knowledge, no study has been performed to examine the effect of coagulation on human blood comparing the difference in properties between pure blood and blood containing standard coagulant agents. This study aims to investigate the effects of coagulation and temperature on the dielectric properties of human blood over a broad frequency range, comparing pure blood to blood mixed with commonly used coagulant and anticoagulant agents. The measurements are performed on freshly extracted human blood samples.

\section{METHODOLOGY}

\subsection{EXPERIMENTAL SET-UP}

Two sets of dielectric measurements were performed at the University of Malta, one to investigate the effects of coagulation over time and the second to investigate the effects of temperature. The measurements were conducted using the commercially available slim form probe from Keysight Technologies over the frequency range of $400 \mathrm{MHz}-20 \mathrm{GHz}$ using 100 frequency points. The $85070 \mathrm{E}$ dielectric probe kit was connected to the Rohde \& Schwarz ZVA50 vector network analyser (VNA). Four blood sample tubes (all standard plastic vacuum bottles) were used for the measurements: one without any added agents (i.e., containing pure whole blood); one with clotting gel and serum separator; and two with anticoagulant agents (EDTA and sodium citrate). These sample tubes were selected since they are the most commonly used in clinics and in blood tests [36], [37]. These sample tubes come with a colored rubber stopper to create a vacuum seal inside of the tube. Each tube has its own standard blood draw volume and is sold with a fixed amount of additive. For example, the standard average amount of EDTA is $0.1 \mathrm{mg}$ for $5 \mathrm{~mL}$ blood. The tubes used in this study all had a maximum volume of $4 \mathrm{~mL}$. The standard sample tube cap has a larger diameter $(13 \mathrm{~mm})$ than the diameter of the probe (2.2 mm). Therefore, an additional customized cap was designed with diameter slightly bigger than the probe; it was placed on the cap of the sample tube to ensure the probe was always in the center of the sample tube. A water bath, specialized beaker and water pump were used to set up an experimental system where measurements were performed using multiple sample tubes (containing the different types of blood samples) all at a constant temperature without moving the coaxial probe and cable. A customized stand was also designed to support the samples inside the beaker. The experimental set-up is shown in Figure 1. A separate experiment was performed to validate the temperature behavior of the system by placing four tubes filled with reference liquid into the beaker. The temperature of each tube was measured every 15 minutes over a period of 5 hours. The measured temperature of each sample was stable over this period, with only a slight variation of $\pm 0.1^{\circ} \mathrm{C}$, thus validating the temperature control system.

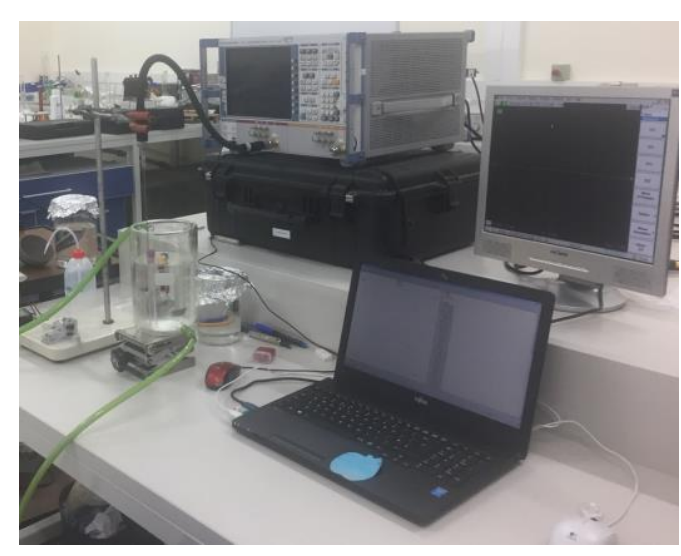

(a)

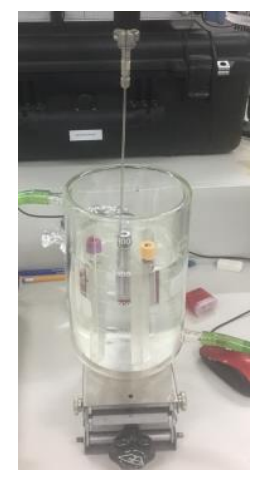

(b)

Figure. 1. Photographs of the experimental set-up a) complete system and b) close-up of probe and the beaker containing the blood sample tubes.

\subsection{BLOOD SAMPLES}

Fresh blood samples from five different volunteers were collected by a doctor, in line with local ethical guidelines. Each blood sample had a volume of $16 \mathrm{~mL}$ and was divided 
into four sub-samples in order to be tested individually. The four sample tubes were filled up to the standard volume with the acquired blood sample and were immediately placed in the beaker with circulating water at $37{ }^{\circ} \mathrm{C}$ to maintain them at body temperature.

\subsection{EXPERIMENTAL MEASUREMENTS}

Four blood samples from different patients, one female and three males, were used to examine the coagulation effects over time. Multiple measurements were performed following the designed measurement protocol with the blood in each of the four sample tubes over a period of five and a half hours.

First, calibration of the dielectric probe measurement system was performed using the standard 3-load calibration method (open, short, deionized water). Subsequent calibrations followed the same procedure. Each calibration was validated by performing reference measurements on $0.9 \% \mathrm{NaCl}$ (physiological saline).

To examine the measurement uncertainty of the system, measurements were performed on another standard reference material. In particular, 15 measurements were performed over three calibrations of the network analyzer on the reference material $0.1 \mathrm{M} \mathrm{NaCl}$ at $22^{\circ} \mathrm{C}$. These measurements were used to calculate the total combined uncertainty (TCU) as in [38]. The TCU was calculated to be 2.1 for relative permittivity, and 3.4 for conductivity, demonstrating that the calibration is both repeatable and accurate. In Figure 2, a set of five calibrations is compared with the reference model of $0.9 \%$ $\mathrm{NaCl}$ from the literature [38], highlighting the repeatability and accuracy of the measurements.

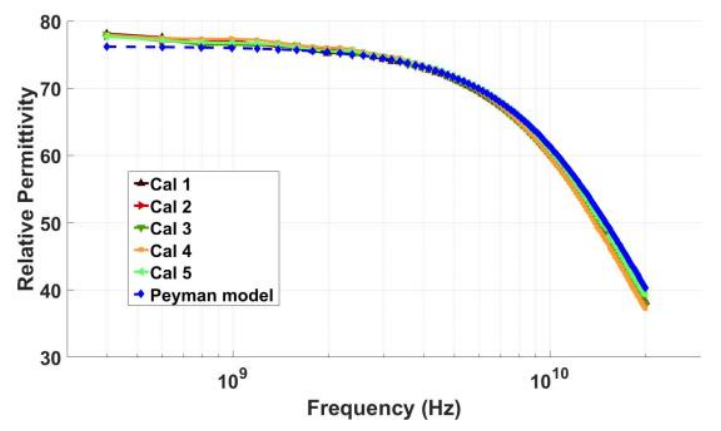

Figure 2. Set of five calibrations performed using $0.9 \% \mathrm{NaCl}$, compared with the reference model of $0.9 \% \mathrm{NaCl}$ from the literature [39].

Following the calibration and validation procedures, measurements were taken on the blood samples. Three measurements were performed at each measurement instance and averaged to obtain one data set. Depending on the additive agent used, the blood may separate into the blood cells and plasma (especially during coagulation). Therefore, for the measurements of pure blood and blood with clotting gel, it was visually ensured that the probe was in contact with the region containing blood cells. A fresh calibration was performed and validated using $0.9 \% \mathrm{NaCl}$ after each set of measurements. The probe was cleaned using alcohol wipes after each measurement. The first measurement was performed as soon as possible and always within 20 minutes of extraction from the body.

A fifth sample from a male volunteer was used to examine the effect of temperature on the dielectric properties of blood. The four tubes were filled with the blood sample and were gradually heated from 37 to $57{ }^{\circ} \mathrm{C}$ in $5{ }^{\circ} \mathrm{C}$ increments. Two sets of measurements were performed at each temperature. At each temperature, calibration was performed and validated using $0.9 \% \mathrm{NaCl}$ solution prior to performing the blood measurements.

\section{Results}

Figure 3 shows the measured relative (real) permittivity of normal whole blood (without added agents) for the first four volunteers. The first measurement recorded from each sample (taken immediately after extraction) is shown in Figure 3a. The mean and standard deviation (STD) of all measurements, over the duration of 5 hours and 36 minutes from the time of extraction, for each sample are calculated and presented in Figure $3 b$. The error bars on each trace represent the STD. The plots show clear variation among the samples, which is expected since the composition of blood and the clotting rates vary naturally from patient to patient. The overall mean shows that the dielectric properties of the female subject's blood are higher than the male subjects, which agrees with other studies that conclude that female blood composition differs from male blood composition [39] and thus, it is reasonable to believe that the dielectric properties would also differ.

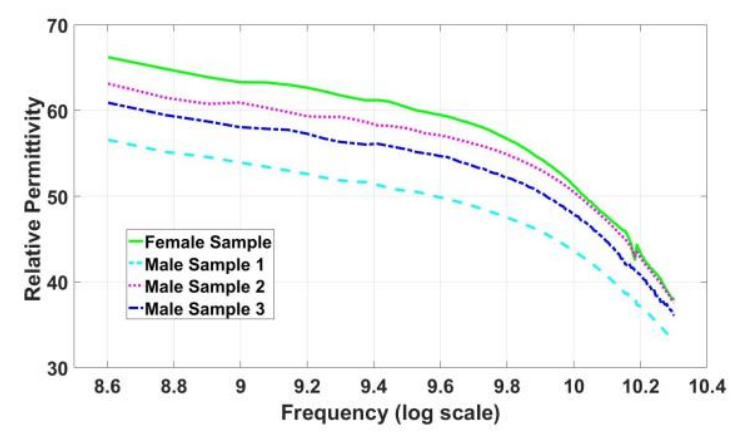

(a) First measurement of all samples

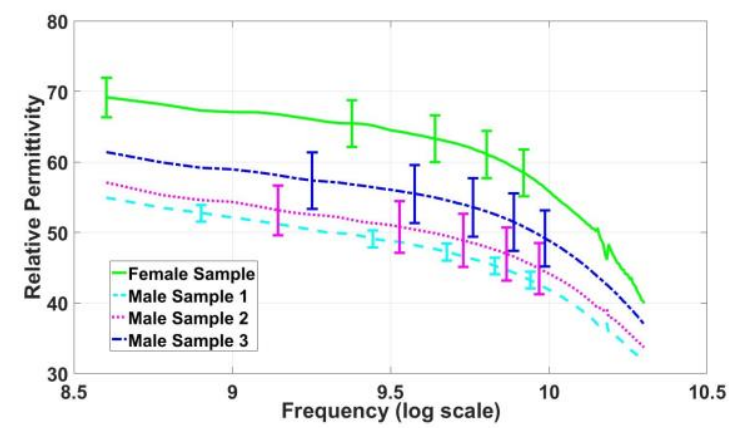

(b) Mean and standard deviation of all measurements for each sample

Figure 3. Real part of pure blood complex permittivity a) first measurement of all samples and b) mean and standard deviation over all measurement times. 
Figure $3 \mathrm{~b}$ also indicates that the dielectric properties change over time differently from sample to sample. For example, the male sample 1 measurements are consistent over time with small STD, whereas the male sample 2 measurements change significantly, by more than 5 points.

Next, the measurements using the tube with clot activator and serum separator are presented in Figure 4. The measurements of male sample 4 were not performed due to an unexpected shortage of the tubes. Figure $4 \mathrm{~b}$ shows that, unlike for normal blood, the mean values of all measurements over time for each sample are closely matched. The female sample has the highest STD relative to the male samples.

The measurements using anticoagulant agents, sodium citrate and EDTA, are shown in Figures 5 and 6, respectively. The first measurement of each sample and mean of all measurements for each sample, with STD as the error bars, using the sodium citrate are shown in Figures $5 \mathrm{a}$ and $5 \mathrm{~b}$, respectively.

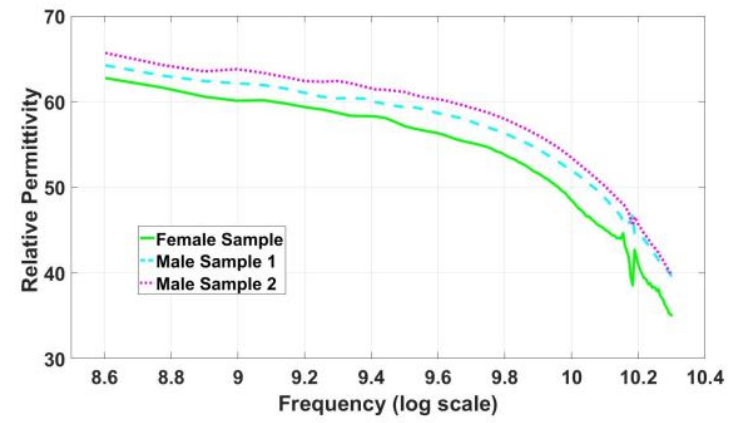

(a) First measurement of all samples

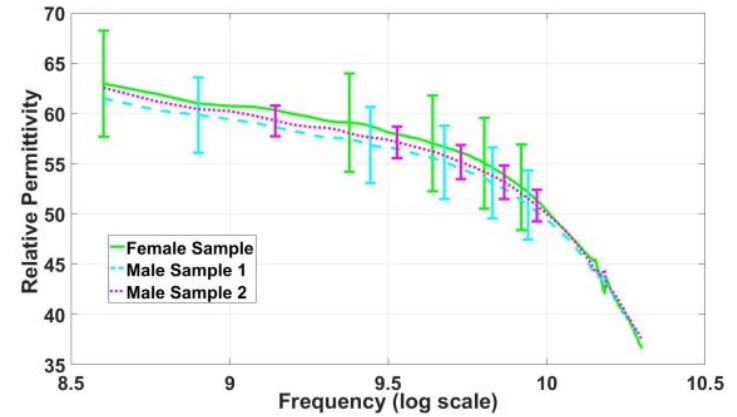

(b) Mean and standard deviation of all measurements for each sample

Figure 4. Real part of complex permittivity of blood with clotting gel and serum separator a) first measurement of all samples and b) mean and standard deviation over all measurement times.

Similarly, the first measurement and mean of all measurements for each sample using EDTA are shown in Figures $6 \mathrm{a}$ and $6 \mathrm{~b}$. The plots again show a clear deviation between the dielectric properties of female sample with the male samples. The dielectric properties of the male blood samples are close to each other, with a standard deviation (STD) of less than $4 \%$ for citrate and less than 3\% for EDTA.

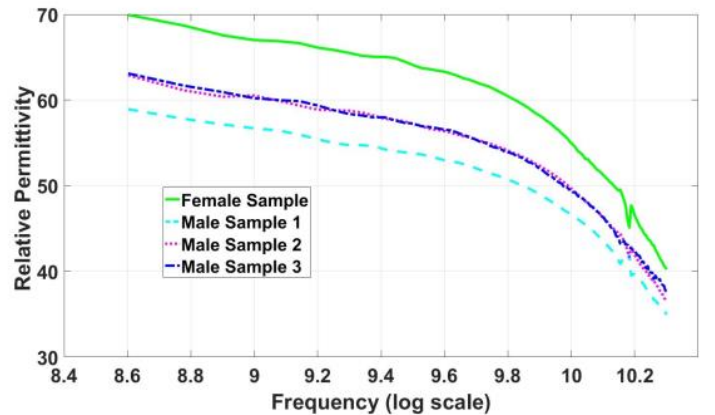

(a) First measurement of all samples

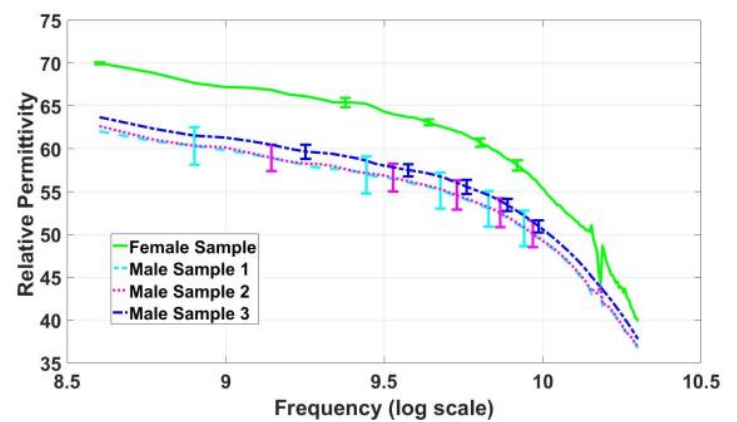

(b) Mean and standard deviation of all measurements for each sample

Figure 5. Real part of complex permittivity of blood with sodium citrate a) first measurement of all samples and b) mean and standard deviation over all measurement times.

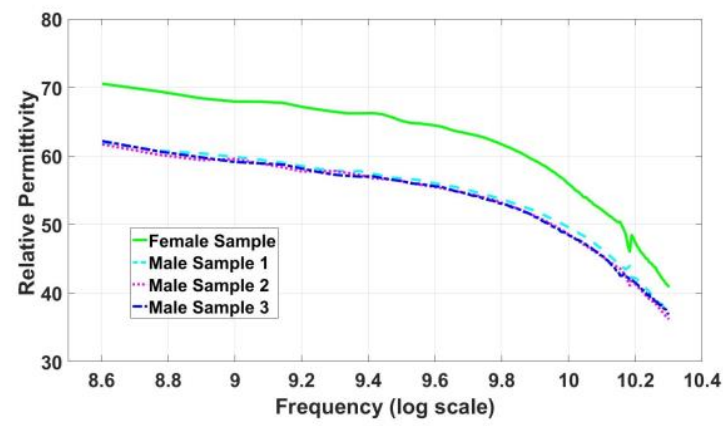

(a) First measurement of all samples

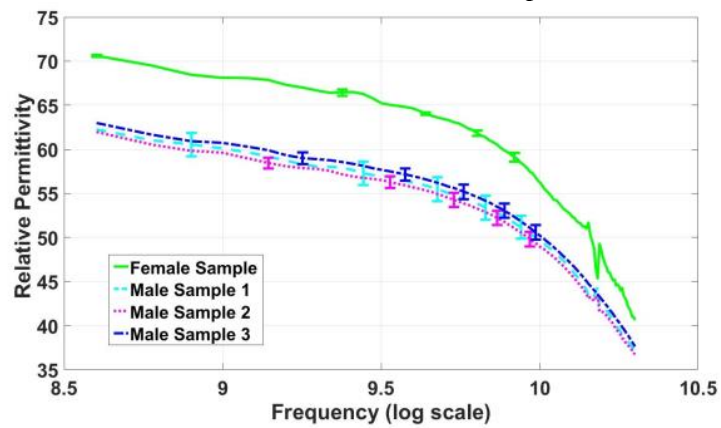

(b) Mean and standard deviation of all measurements for each sample

Figure 6. Real part of complex permittivity of blood with EDTA a) first measurement of all samples and b) mean and standard deviation over all measurement times.

The female sample differs from the male samples by $11-13 \%$. The measurements also indicate that the difference between female and male blood is more than the difference between two or more male blood samples. One reason for this discrepancy is the level of coagulation of the blood - in 
particular, the female blood was visibly not coagulating over time whereas the male samples were thickening. Similar trends have been found for the imaginary part.

The mean and STD values of relative permittivity and conductivity at the start and end frequencies for all measurements using each type of tube are summarized in Tables 1 and 2. It is found that the standard deviation in the measurements of pure blood samples (without any added agents) is as large as $11 \%$, whereas the standard deviation in the blood in EDTA anticoagulant is as little as $1.31 \%$ which is within the measurement uncertainty. These results suggest that the dielectric properties of blood held in tubes containing coagulant or anticoagulant agents may not match well with those of pure, untreated blood on a patient to patient basis. The maximum difference, across all patients, between the pure blood and the treated blood measurements at $400 \mathrm{MHz}$ is 13.8 permittivity points and at $20 \mathrm{GHz}$ is 7.1 permittivity points.

Table 1. Statistical information of relative permittivity for measurements across all samples using each type of sample tube.

\begin{tabular}{l|l|cccc}
\hline \multicolumn{2}{c}{ Frequency } & \begin{tabular}{c} 
No \\
\multicolumn{2}{c}{ additives }
\end{tabular} & $\begin{array}{c}\text { Clot } \\
\text { activator }\end{array}$ & $\begin{array}{c}\text { Blood with } \\
\text { Sodium } \\
\text { Citrate }\end{array}$ & EDTA \\
\hline \multirow{2}{*}{ Mean } & $400 \mathrm{MHz}$ & 61.99 & 62.50 & 63.44 & 63.10 \\
\cline { 2 - 6 } & $20 \mathrm{GHz}$ & 36.32 & 37.06 & 37.40 & 37.41 \\
\hline \multirow{2}{*}{ STD } & $400 \mathrm{MHz}$ & $10.40 \%$ & $6.32 \%$ & $3.90 \%$ & $3.90 \%$ \\
\cline { 2 - 6 } & $20 \mathrm{GHz}$ & $10.73 \%$ & $6.44 \%$ & $3.30 \%$ & $3.40 \%$ \\
\hline \multirow{2}{*}{ Range } & $400 \mathrm{MHz}$ & $53.8-72.8$ & $49.9-68.1$ & $58.9-70.1$ & $61.2-70.7$ \\
\cline { 2 - 6 } & $20 \mathrm{GHz}$ & $30.8-42.1$ & $30.4-40.3$ & $35-40.2$ & $35.8-40.9$ \\
\hline
\end{tabular}

Table 2. Statistical information of conductivity for measurements across all samples using each type of sample tube.

\begin{tabular}{l|l|cccc}
\hline \multicolumn{2}{c}{ Frequency } & \begin{tabular}{c} 
No \\
\multicolumn{2}{c}{ additives }
\end{tabular} & $\begin{array}{c}\text { Blot } \\
\text { activator }\end{array}$ & $\begin{array}{c}\text { Sod with } \\
\text { Sodium } \\
\text { Citrate }\end{array}$ & EDTA \\
\hline \multirow{2}{*}{ Mean } & $400 \mathrm{MHz}$ & 1.27 & 1.32 & 1.35 & 1.39 \\
\cline { 2 - 6 } & $20 \mathrm{GHz}$ & 29.85 & 30.39 & 29.74 & 29.65 \\
\hline \multirow{2}{*}{ STD } & $400 \mathrm{MHz}$ & $18.70 \%$ & $7.57 \%$ & $7.04 \%$ & $6.99 \%$ \\
\cline { 2 - 6 } & $20 \mathrm{GHz}$ & $20.48 \%$ & $9.44 \%$ & $9.91 \%$ & $9.70 \%$ \\
\hline \multirow{2}{*}{ Range } & $400 \mathrm{MHz}$ & $0.95-1.66$ & $1.08-1.48$ & $1.22-1.6$ & $1.31-1.68$ \\
\cline { 2 - 6 } & $20 \mathrm{GHz}$ & $22.2-41.6$ & $24.6-34.8$ & $25.6-38.7$ & $25.5-38.6$ \\
\hline
\end{tabular}

Lastly, one male blood sample was used to examine the effect of changing temperature on the dielectric properties of blood. This effect is particularly important for hyperthermia and ablation applications. The sample was heated in increments of $5^{\circ} \mathrm{C}$. No clear trend with temperature was found for the pure blood, likely due to the variations in clotting. It was visually observed (through the thickening of the sample) that clotting had begun prior to the first set of measurements at body temperature; then as the sample heated the clotting seemed to reduce, before increasing again at higher temperatures. However, this lack of trend was not evident in treated blood samples. The real part of complex permittivity of blood samples with anticoagulant agents consistently decreases with increasing temperature, up to the cross-over frequency. The results are in good agreement with [1]. As an example, the results of heated blood sample with EDTA are shown in Figure 7.

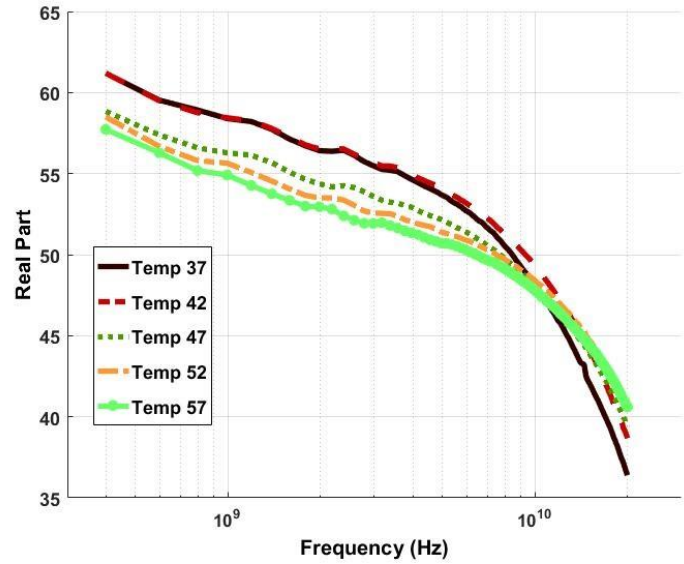

(a) Real Part of complex permittivity

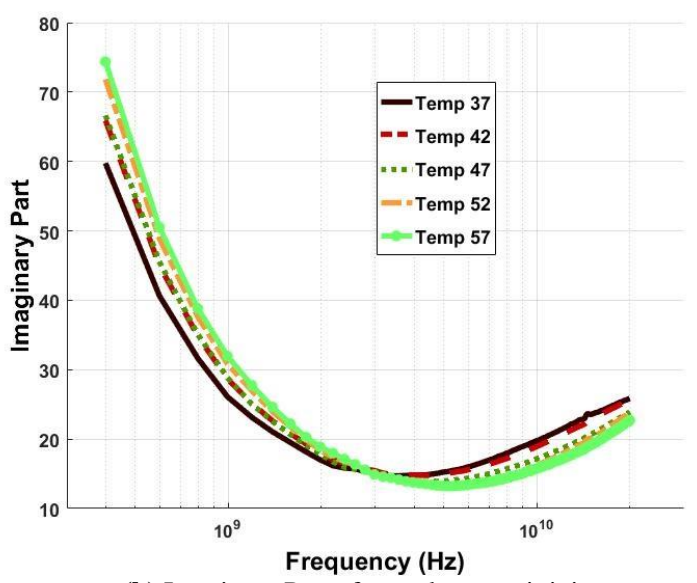

(b) Imaginary Part of complex permittivity

Figure 7. Real and imaginary parts of complex permittivity of blood with EDTA with increasing temperature (the temperature values are in degrees Celsius).

\section{CONCLUSION}

In this paper, dielectric measurements of human blood were performed to investigate the effects of coagulation and temperature over time using four different blood sample tubes: one untreated containing pure whole blood, two with standard anticoagulant agents, and one with clot activator and serum separator. Four freshly extracted blood samples were used to examine the effect of coagulation on the dielectric properties over time for a frequency range of $400 \mathrm{MHz}-20 \mathrm{GHz}$, while pure blood sample was used to examine the effect of temperature on the dielectric properties of blood. The results have demonstrated that the pure blood has no clear trends in coagulation or temperature over time due to various patient dependent factors such as clotting rate, composition of blood, and hematocrit levels. Notably, the dielectric properties of pure blood differ significantly from those of blood with anticoagulant agent for some patients, even for measurements conducted on the same blood sample at the same time. This finding is significant as it suggests that blood dielectric measurements conducted on biobank or blood bank samples may not be representative of the natural, in-vivo variation in blood properties. The dielectric properties of male blood 
samples are similar to each other but are clearly separated from the female blood sample. The relative permittivity of blood with anticoagulant agents decreases with increasing temperature, up to the crossover frequency around $10 \mathrm{GHz}$, while the imaginary part of the permittivity increases with increasing temperature up to a crossover point of $\sim 3 \mathrm{GHz}$. This work suggests that a comprehensive investigation of the blood dielectric properties with a large number of samples is required to carefully investigate all confounders. As such, this study provides the groundwork for future studies to examine all aspects of the dielectric properties of human blood that have not been addressed before, and to build a comprehensive database of the properties and their dependencies.

\section{ACKNOWLEDGMENTS}

The research leading to these results has received funding from the European Research Council under the ERC Grant Agreement n. 637780: 'BIOELECPRO' and the Irish Research Council (grant numbers RCS1325 and RCS1377). This work has been developed in the framework of COST Action MiMed (TD1301).

\section{REFERENCES}

[1] M. Wolf, R. Gulich, P. Lunkenheimer, and a Loidl, "Broadband dielectric spectroscopy on human blood.," Biochim. Biophys. Acta, vol. 1810, no. 8, pp. 727-40, 2011.

[2] F. F. Becker, X. B. Wang, Y. Huang, R. Pethig, J. Vykoukal, and P. R. Gascoyne, "Separation of human breast cancer cells from blood by differential dielectric affinity.," Proc. Natl. Acad. Sci., vol. 92, no. 3, pp. 860-864, 1995.

[3] Y. Hayashi, Y. Katsumoto, I. Oshige, S. Omori, A. Yasuda, and K. Asami, "Dielectric inspection of erythrocytes," J. Non. Cryst. Solids, vol. 356, no. 11-17, pp. 757-762, 2010.

[4] Y. Hayashi, M. A. Brun, K. Machida, and M. Nagasawa, "Principles of Dielectric Blood Coagulometry as a Comprehensive Coagulation Test," Anal. Chem., vol. 87, no. 19, pp. 10072-10079, 2015.

[5] Y. Hayashi, Y. Katsumoto, S. Omori, A. Yasuda, K. Asami, M. Kaibara, and I. Uchimura, "Dielectric coagulometry: A new approach to estimate venous thrombosis risk," Anal. Chem., vol. 82, no. 23, pp. 9769-9774, 2010.

[6] W. H. Geerts, D. Bergqvist, G. F. Pineo, J. A. Heit, C. M. Samama, M. R. Lassen, and C. W. Colwell, "Prevention of Venous Thromboembolism*: American College of Chest Physicians Evidence-Based Clinical Practice Guidelines (8th Edition).," Chest, vol. 133, no. 6, p. 381S--453S, 2008.

[7] A. Trakic, M. Akhand, H. Wang, D. Mason, F. Liu, S. Wilson, and S. Crozier, "Computational modelling of blood-flow-induced changes in blood electrical conductivity and its contribution to the impedance cardiogram," Physiol. Meas., vol. 31, no. 1, p. 13, 2009.

[8] O. Baskurt, M. Uyuklu, and H. Meiselman, "Simultaneous monitoring of electrical conductance and light transmittance during red blood cell aggregation," Biorheology, vol. 46, no. 3, pp. 239249, 2009.

[9] A. Andreano and C. L. Brace, "A comparison of direct heating during radiofrequency and microwave ablation in ex vivo liver," Cardiovasc. Intervent. Radiol., vol. 36, no. 2, pp. 505-511, 2013.

[10] L. Farina, N. Weiss, Y. Nissenbaum, M. Cavagnaro, V. Lopresto, R. Pinto, N. Tosoratti, C. Amabile, S. Assarino, and S. N. Goldberg, "Characterisation of tissue shrinkage during microwave thermal ablation," Int. J. Hyperth., vol. 30, no. 7, pp. 419-428, 2014.

[11] X. Nie, Q. Nan, X. Guo, and Z. Tian, "Numerical study of the effect of blood vessel on the microwave ablation shape," Biomed. Mater. Eng., vol. 26, no. s1, pp. S265-S270, 2015.

[12] P. T. Nguyen, A. Abbosh, and S. Crozier, "Microwave Hyperthermia for Breast Cancer Treatment Using Electromagnetic and Thermal Focusing Tested on Realistic Breast Models and Antenna Arrays," IEEE Trans. Antennas Propag., vol. 63, no. 10, pp. 4426-4434, 2015.

[13] T. Sherertz and C. J. Diederich, "Hyperthermia in Locally Recurrent Breast Cancer," in Radiation Therapy Techniques and Treatment Planning for Breast Cancer, United States: Springer, 2016, pp. 145158.

[14] P. T. Nguyen, "Focusing microwave hyperthermia in realistic environment for breast cancer treatment," University of Queensland, Australia, 2016.

[15] ICNIRP, "Guidelines for limiting exposure to time-varying electric, magnetic, and electromagnetic fields (up to $300 \mathrm{GHz}$ )," Heal. Phys, vol. 74, no. 4, pp. 494-522, 1998.

[16] IEEE Standards Coordinating Committee, IEEE Standard for Safety Levels with Respect to Human Exposure to Radio Frequency Electromagnetic Fields, 3kHz to $300 \mathrm{GHz} .1992$.

[17] B. B. Beard, W. Kainz, T. Onishi, T. Iyama, S. Watanabe, O. Fujiwara, J. Wang, G. Bit-Babik, A. Faraone, and J. Wiart, "Comparisons of computed mobile phone induced SAR in the SAM phantom to that in anatomically correct models of the human head," IEEE Trans. Electromagn. Compat., vol. 48, no. 2, pp. 397-407, 2006.

[18] H. Pauly and H. Schwan, "Dielectric properties and ion mobility in erythrocytes," Biophys. J., vol. 6, no. 5, p. 621, 1966.

[19] J. Krupa, K. B, and J. Terlecki, "Method of calculating conductivity of human erythrocytes interior based on measurement of electric magnitudes of suspension," Biophysik, vol. 8, no. 3, p. 227, 1972.

[20] R. Pethig, "Dielectric Properties of Biological Materials : Biophysical and Medical Applications," IEEE Trans. Electr. Insul., vol. EI-19, no. 5, pp. 453-474, 1984.

[21] J. M. Alison and R. J. Sheppard, "Dielectric properties of human blood at microwave frequencies.," Phys. Med. Biol., vol. 38, no. 7, pp. 971-978, 1993.

[22] Y. Feldman, I. Ermolina, and Y. Hayashi, "Time domain dielectric spectroscopy study of biological systems," IEEE Trans. Dielectr. Electr. Insul., vol. 10, no. 5, pp. 728-753, 2003.

[23] H. Schwan, "Electrical properties of blood and its constitutents: Alternating current spectroscopy," Blut, vol. 46, no. 4, pp. 185-197, 1983.

[24] T.-X. Zhao, "Electrical impedance and haematocrit of human blood with various anticoagulants," Physiol. Meas., vol. 14, no. 3, p. 299, 1993.

[25] H. Beving, L. Eriksson, C. Davey, and D. Kell, "Dielectric properties of human blood and erythrocytes at radio frequencies $(0.2--10 \mathrm{MHz})$; dependence on cell volume fraction and medium composition," Eur. Biophys. J., vol. 23, no. 3, pp. 207-215, 1994.

[26] J.-Z. Bao, C. C. Davis, and M. L. Swicord, "Microwave dielectric measurements of erythrocyte suspensions," Biophys. J., vol. 66, no. 3, p. 2173, 1994.

[27] F. Bordi, C. Cametti, R. Misasi, R. De Persio, and G. Zimatore, "Conductometric properties of human erythrocyte membranes: dependence on haematocrit and alkali metal ions of the suspending medium," Eur. Biophys. J., vol. 26, no. 3, pp. 215-225, 1997.

[28] R. Lisin, B. Z. Ginzburg, M. Schlesinger, and Y. Feldman, "Time domain dielectric spectroscopy study of human cells. I. Erythrocytes and ghosts," Biochim. Biophys. Acta (BBA)-Biomembranes, vol. 1280, no. 1, pp. 34-40, 1996.

[29] T. Chelidze, "Dielectric spectroscopy of blood," J. Non. Cryst. Solids, vol. 305, no. 1-3, pp. 285-294, 2002.

[30] F. Jaspard, M. Nadi, and a Rouane, "Dielectric properties of blood: an investigation of haematocrit dependence.," Physiol. Meas., vol. 24, no. 1, pp. 137-147, 2003.

[31] Y. Hayashi, I. Oshige, Y. Katsumoto, S. Omori, A. Yasuda, and K. Asami, "Dielectric inspection of erythrocyte morphology," Phys. Med. Biol., vol. 53, no. 10, p. 2553, 2008.

[32] Y. Zhang, L. Zhong, S. Tan, C. Xu, A. Preparation, and C. Suspension, "Dielectric Properties of Red Blood Cell Suspensions Based on Broadband Dielectric Spectrum," Physics (College. Park. Md)., no. 50277030, pp. 3-6, 2010.

[33] S. Abdalla, S. S. Al-ameer, and S. H. Al-Magaishi, "Electrical properties with relaxation through human blood," Biomicrofluidics, vol. 4 , no. 3, pp. 1-16, 2010.

[34] A. Rauf, "a Dielectric Study on Human Blood and Plasma," Int. J. Sci. Environ. Technol., vol. 2, no. 6, pp. 1396-1400, 2013.

[35] S. Gabriel, C. Gabriel, and R. W. Lau, "The dielectric properties of biological tissues: III. Parametric models for the dielectric spectrum 
of tissues.," Phys. Med. Biol., vol. 41, no. 11, pp. 2271-2293, 1996. BioBank UK, Blood Sample Collection, Processing and Transport. 2011.

[37] P. Elliott and T. C. Peakman, "The UK Biobank sample handling and storage protocol for the collection, processing and archiving of human blood and urine," Int. J. Epidemiol., vol. 37, no. 2, pp. 234 244,2008

[38] C. Gabriel and A. Peyman, "Dielectric measurement: error analysis and assessment of uncertainty.," Phys. Med. Biol., vol. 51, no. 23, pp. 6033-6046, 2006.

[39] M. Kameneva, M. Watach, and H. Borovetz, "Gender difference in rheologic properties of blood and risk of cardiovascular diseases," Clin. Hemorheol. Microcirc., vol. 21, no. 3, 4, pp. 357-363, 1999.

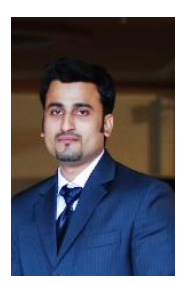

Mr. Saqib Salahuddin was born in Lahore, Pakistan in 1985. He received the BSc degree in computer engineering from COMSATS Institute of Information Technology, Abbottabad, Pakistan (with High Honours) in 2007 and the MSc degree in microelectronics systems design from the University of Southampton, United Kingdom in 2009. He is currently working towards the PhD (Electrical \& Electronics) at the National University of Ireland, Galway, Ireland. Mr. Saqib Salahuddin is a member of COST (European Cooperation in Science and Technology) Action MiMED and COST Action EMF-MED. He was Lecturer in Electrical Engineering Department at COMSATS Institute of Information Technology, Lahore, Pakistan from 2007 to 2014 and a research member of COMVIS (Computer Vision Research Group) from 2011 to 2014. His research interests include Medical Systems, Embedded Systems, Optimization Algorithms, and Electromagnetics.

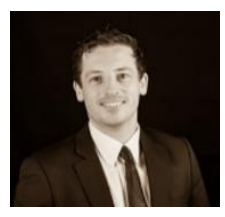

Dr. Martin O'Halloran received a B.Eng. (Hons.) and $\mathrm{PhD}$. in electrical engineering from the National University of Ireland Galway (NUIG) in 2004 and 2009, respectively. He also holds an MSc. In clinical research (2014), also from NUIG. Dr. O'Halloran is the Director of the Translational Medical Device Lab at NUIG, and is Director of Enabling Technologies at BioInnovate (an affiliate of Stanford's BioDesign Programme). He has over 25 national and international awards, including Engineers Ireland Engineer of the Year 2014, and Science Foundation Ireland's EC Researcher of the Year 2016. Dr. O'Halloran's research is funded by Science Foundation Ireland, the Irish Research Council and the European Research Council. His research is focused on patient-centered medical device design and development.

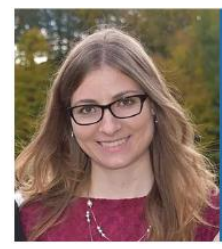

Emily Porter (S'11-M'15) received the B.Eng., M.Eng., and Ph.D. degrees in electrical engineering from McGill University, Montreal, Canada, in 2009, 2010, and 2015, respectively. Dr. Porter was a recipient of the 2013 IEEE Antennas and Propagation Society Doctoral Research Award for her work on breast health monitoring using a time-domain microwave system. Since 2015 , she has been a postdoctoral researcher with the Translational Medical Device Laboratory at the National University of Ireland Galway. Her current research interests include the measurement of dielectric properties of biological tissues and the development novel technologies for therapeutic and diagnostic applications of electromagnetic waves.

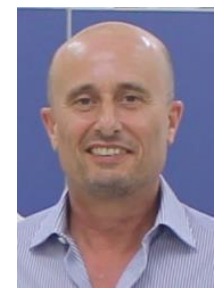

Dr. Charles Sammut holds a BA in education from UM (1980), a BSc in physics with Physical Electronics (First Class Honours) (1987) and a $\mathrm{PhD}$ in the field of microwave semiconductor devices (1992) from the University of Bath, UK. He Joined the University of Malta in 1987 and was awarded a Commonwealth Academic Staff Scholarship to read for the PhD degree, for which he was awarded the Deryck Chesterman Medal from Bath University. He is currently full Professor and
Head of the Department of Physics, Dean of the Faculty of Science and leads the Electromagnetics Research Group (EMRG). His current research interests include: dielectric spectroscopy of biological tissues for medical applications; computational electromagnetics; antenna design; exposure of workers and the general public to non-ionising electromagnetic fields; biological effects of non-ionising electromagnetic fields. Professor Sammut is also a Management Committee Member of five COST Actions: MP 1204, TD 1301, BM 1205, BM 1309 and IC 1407.

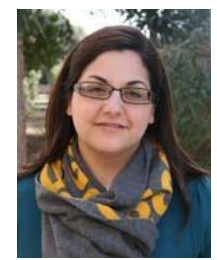

Lourdes Farrugia received a B.Sc. (Hons.) in mathematics and physics and a M. Sc in physics from the University of Malta in 2008 and 2009, respectively. She joined the Physics department in 2011 as a Research Officer working in an Electromagnetics laboratory funded by the European Regional Development Fund (ERDF). She obtained a Ph.D. from the University of Malta in 2016. Her research interests are mainly focused on aspects of instrumentation and measurement of physical quantities, especially sensor design, applied electromagnetics (in particular, dielectric properties of biological tissue), electromagnetic compatibility, and biological effects of electromagnetic radiation.

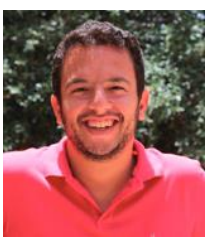

Mr. Julian Bonello read for a BSc in physics and computer information systems (C.I.S.) from the University of Malta (UM) and an MSc in physics. Currently, he is reading a $\mathrm{PhD}$ in bioelectromagnetics, with the Physics department at the UM. Julian has recently joined the Physics department as a Systems Engineer within the Electromagnetics laboratory. His research interests are occupational and general public exposure to non-ionising electromagnetic fields, dielectric properties of biological tissues, and computational electromagnetics.

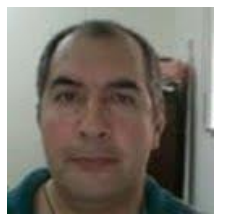

Pierre Schembri-Wismayer worked in St Luke's teaching hospital in the department of Accident and Emergency medicine as well as in the National Blood Transfusion centre before joining the University of Malta's Dept. of Anatomy. He also worked part time for a biotech company called Atheneum biotechnology. He read for his undergraduate degree in Medicine and Surgery at the University of Malta and after two years of internship and having received his warrant, he did some voluntary medical work in Kenya and then left to pursue his $\mathrm{PhD}$ in molecular oncology at the Beatson Institute for Cancer Research in association with Glasgow University. Prof SchembriWismayer won a contract with the EU's FP6 program, to produce an international television series, called X-lab, broadcast in Malta and Cyprus, to encourage youngsters to take part in science and possibly take it up as a career through highlighting local scientific heroes and job opportunities. Prof Schembri-Wismayer regularly talks locally about cord blood stem cell banking and was for a long time the Programme committee expert for the Malta council of science and technology (MCST) in relation to the Life sciences. Research into cancer immunotherapy, cancer differentiation, stem cells and biomechanics are Prof Schembri-Wismayer's interest. He also teaches undergraduate students both in the Faculty of Medicine and Surgery as well as in the Faculty of Health sciences. 